\title{
Paquimeningite hipertrófica idiopática espinhal: Relato de caso
}

\section{Idiopathic Hypertrophic Spinal Pachymeningitis: A Case Report}

\author{
Lucas Lodomiro Araújo Melo ${ }^{1,2}$ Murilo Tavares Daher ${ }^{1,2}$ Marcus Vinícius Magno Gonçalves ${ }^{3}$
}

Marianne Borges Freitas 4 (1)

${ }^{1}$ Faculdade de Medicina, Departamento de Ortopedia, Universidade

Federal de Goiás, Goiânia, GO, Brasil

Endereço para correspondência Lucas Lodomiro Araújo Melo, MD,

${ }^{2}$ Centro de Reabilitação e Readaptação Henrique Santillo (CRER), Ortopedista, Brasil (e-mail: lucaslodomiro@yahoo.com.br).

Goiânia, GO, Brasil

${ }^{3}$ Departamento de Medicina, Universidade Regional de Joinville

(UNIVILLE), Joinville, SC, Brasil

${ }^{4}$ Universidade Federal de Uberlândia, Uberlândia, MG, Brazil

Rev Bras Ortop 2022;57(3):521-523.

\section{Resumo \\ Palavras-chave \\ - medula espinal \\ - meningite/patologia \\ - meningite/ fisiopatologia \\ - hipertrofia}

A paquimeningite hipertrófica idiopática é uma causa rara de sintomas neurológicos apresentando mielopatia por compressão da medula espinhal. Relatamos um caso de paquimeningite com manifestação primária de tetraparesia após trauma de baixa energia e recorrência dos sintomas de mielopatia 5 anos após a cirurgia. A paciente, uma mulher de 19 anos, foi submetida a extensa investigação sem evidências de qualquer doença de base. Uma biópsia da meninge revelou processo inflamatório inespecífico com extensa fibrose da dura máter, também visualizado no período perioperatório. Esses achados, associados à exclusão de outras causas, sugerem o diagnóstico de paquimeningite hipertrófica idiopática.

Idiopathic hypertrophic pachymeningitis is rare cause of neurological symptoms with myelopathy due to spinal cord compression. We report a case of pachymeningitis, which was manifested primarily by tetraparesis after low-energy trauma and recurrence the myelopathy symptoms after 5 years of surgery. The patient, a 19-year-old woman, was subjected to extensive investigation without evidence of any underlying disease. A meningeal biopsy was performed and showed an unspecific inflammatory process with extensive fibrosis of the dura mater. These findings, associated with the exclusion of other causes, suggest idiopathic hypertrophic pachymeningitis.

\section{Introdução}

A paquimeningite hipertrófica idiopática espinhal (PHIE) é uma doença inflamatória autoimune rara que leva à compres- são da medula espinhal. A PHIE, que pode estar relacionada à IgG4, é um distúrbio imunomediado caracterizado por lesões decorrentes de uma reação inflamatória inespecífica associada à fibrose e infiltração linfoplasmocítica que compõe o espectro recebido

12 de Outubro de 2020

aceito

01 de Dezembro de 2020

Publicado on-line

Agosto 13, 2021
DOI https://doi.org/

$10.1055 / \mathrm{s}-0041-1724087$. ISSN 0102-3616. (c) 2021. Sociedade Brasileira de Ortopedia e Traumatologia. All rights reserved.

This is an open access article published by Thieme under the terms of the Creative Commons Attribution-NonDerivative-NonCommercial-License, permitting copying and reproduction so long as the original work is given appropriate credit. Contents may not be used for commercial purposes, or adapted, remixed, transformed or built upon. (https://creativecommons.org/ licenses/by-nc-nd/4.0/)

Thieme Revinter Publicações Ltda., Rua do Matoso 170, Rio de Janeiro, RJ, CEP 20270-135, Brazil 
das doenças linfoproliferativas. Apresentamos um caso com esta patologia e os achados intraoperatórios, exames de imagem (ressonância magnética) e biópsia da meninge.

\section{Relato de Caso}

Uma mulher de 19 anos apresentou tetraparesia aguda após trauma cervical do "tipo chicote". O primeiro exame de imagem realizado (-Figura $\mathbf{1}$ ) revelou sinais de estenose cervical nos níveis $\mathrm{C} 3$ a C6, sugestivos de hematoma peridural no diagnóstico diferencial. A princípio, uma laminoplastia de C3 a C6 foi realizada. Após a cirurgia, não houve melhora do estado neurológico, e a imagem pós-operatória ainda mostrava estenose cervical. Com isso, a paciente foi submetida a uma corpectomia de $\mathrm{C} 3$ aliado com artrodese cervical anterior dos níveis de C2 a C4 (-Figura 2).

A paciente apresentou melhora motora e sensorial progressiva após a cirurgia e a fisioterapia. Cinco anos depois, a paciente sofreu piora abrupta da marcha sem correlação com evento traumático e piora do déficit motor nos membros superiores e inferiores (assimétrica, com maior gravidade do lado direito). A ressonância magnética revelou estenose cervicotorácica de C7 a T4 (-Figura 3), com indicação de laminoplastia dos níveis C7 a T4. Durante a cirurgia, um espessamento posterior da meninge foi observado (- Figura 4), o que levou à decisão de fazer uma duraplastia, com o uso de um substituto de dura máter, aliado com artrodese posterior de C7 a T5 (- Figura 5). O laudo de biópsia da meninge coletada no intraoperatório crônico inespecífico, com infiltrado linfoplasmocítico sugestivo de paquimeningite hipertrófica (-Figura 6). A paciente evoluiu com melhora da marcha e dos sintomas motores após a cirurgia. As causas secundárias foram excluídas durante a investigação clínica e o diagnóstico clínico-histológico foi PHIE.

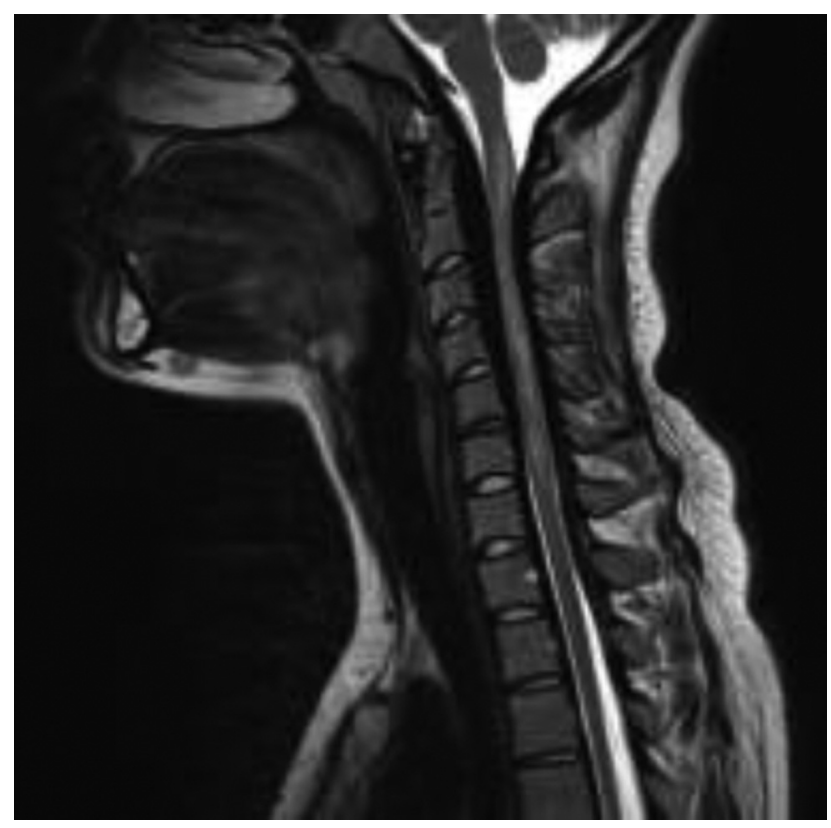

Fig. 1 Imagem de ressonância magnética à internação com estenose cervical de múltiplos níveis associada a sinais de mielopatia.

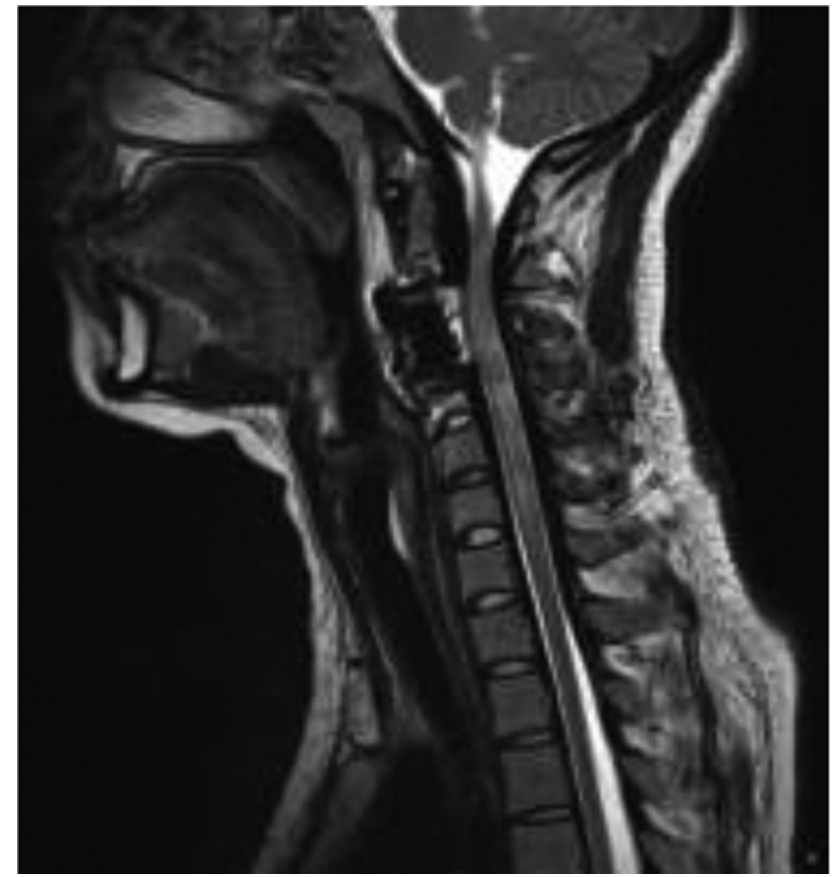

Fig. 2 Imagem de ressonância magnética após corpectomia em C3, artrodese anterior em C2 a C4 e laminoplastia em C3 a C6.

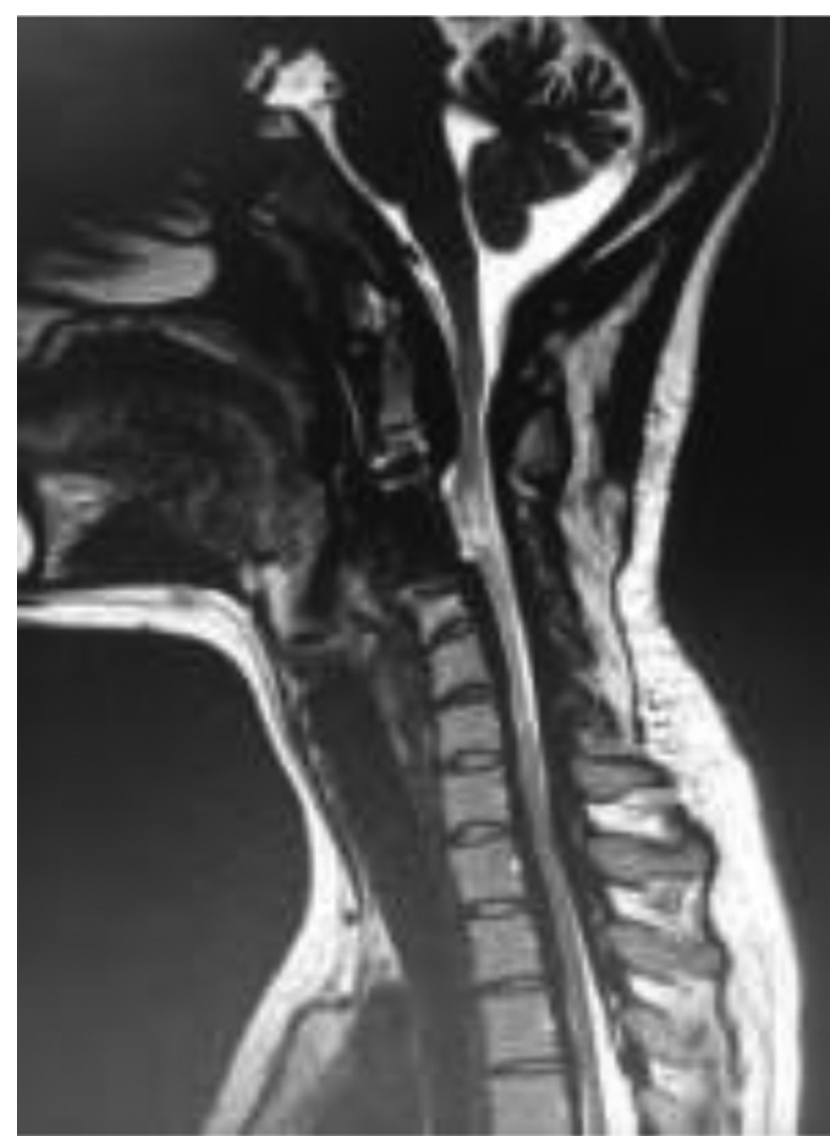

Fig. 3 Estenose cervicotorácica.

\section{Discussão}

A paquimeningite hipertrófica é uma doença rara causada por uma inflamação crônica que evolui para a fibrose da dura- 


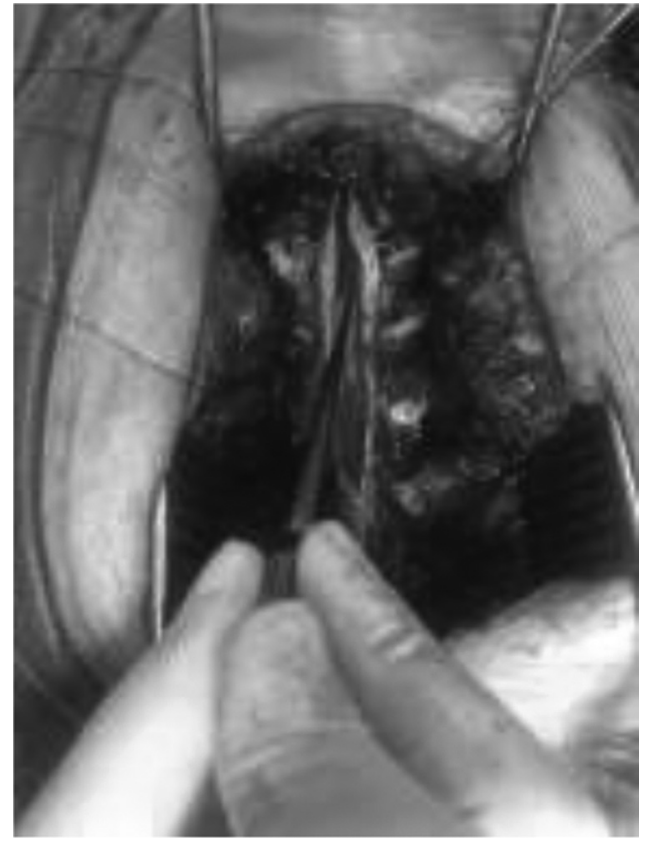

Fig. 4 Hipertrofia da meninge após durotomia.

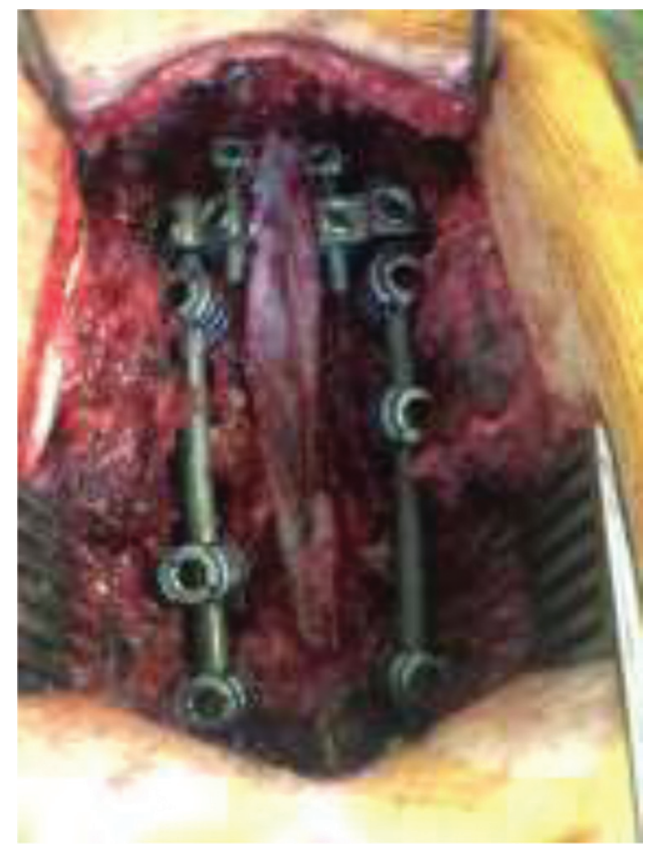

Fig. 5 Aspecto final após duroplastia com artrodese posterior em C7 a T5.

máter. ${ }^{1,2}$ Pode ser idiopática ou secundária a doenças sistêmicas, como sarcoidose, tuberculose, meningite fúngica, artrite reumatoide, carcinomatose e outras doenças inflamatórias

Esta doença pode ser intracraniana ou espinhal. Uma forma espinhal, a PHIE, é extremamente rara e pode estar associada à mielopatia e/ou radiculopatia. A PHIE pertence ao espectro de doenças relacionadas à IgG4, caracterizada por fibrose difusa combinada à infiltração linfoplasmocítica rica em plasmócitos teciduais IgG4- positivos. ${ }^{3}$ A estenose do canal medular pode ser responsável pelo agravamento neurológico abrupto após o trauma. À ressonância magnética, é comum observar espessamento e hipointensidade da dura-máter nas ponderações em

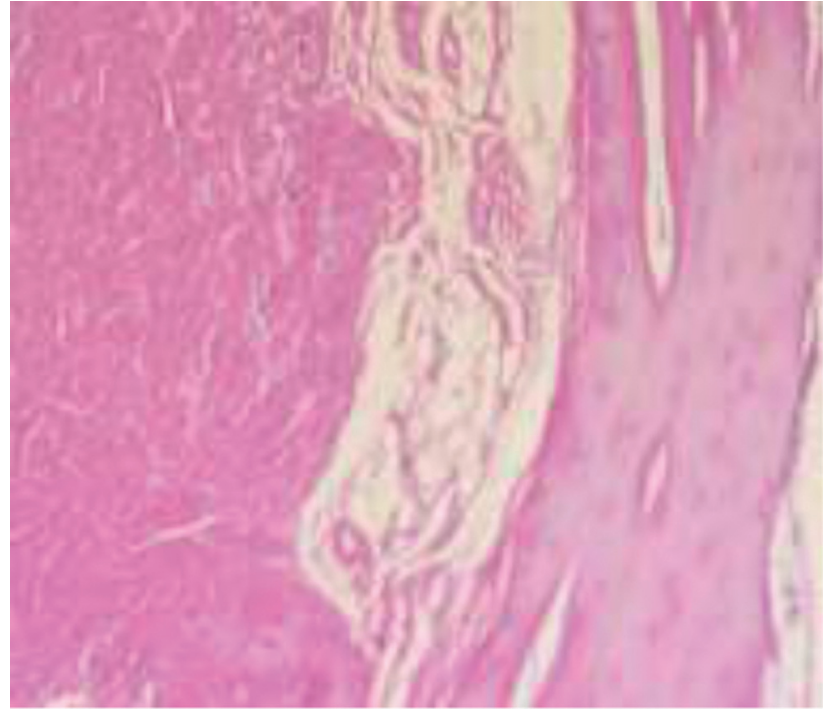

Fig. 6 Processo inflamatório crônico inespecífico das meninges, com infiltrado de linfoplasmocítico.

T1 e T2, com forte realce em múltiplos níveis. ${ }^{4,5} \mathrm{O}$ tratamento clínico inclui o uso prolongado de medicamentos imunossupressores, como os corticosteroides. O tratamento cirúrgico é realizado em pacientes com mielopatia ou radiculopatia associada a alterações neurológicas e é indicado para descompressão do canal medular. Em alguns casos, uma duraplastia é necessária para descompressão adequada.

\section{Conclusão}

A paquimeningite hipertrófica é uma doença rara que pode causar estenose do canal espinhal e sinais de mielopatia. Nestes casos, a descompressão cirúrgica pode ser uma das primeiras opções terapêuticas e, aliada ao uso de imunossupressores, pode reduzir as sequelas neurológicas desses pacientes.

\section{Suporte Financeiro}

Não houve suporte financeiro de fontes públicas, comerciais, ou sem fins lucrativos.

Conflito de Interesses

Os autores não têm conflito de interesses a declarar.

\section{Referências}

1 de Deus-Silva L, Queiroz LS, Zanardi Vd VA, et al. Hypertrophic pachymeningitis: case report. Arq Neuropsiquiatr 2003;61(01): 107-111

2 Rossi S, Giannini F, Cerase A, et al. Uncommon findings in idiopathic hypertrophic cranial pachymeningitis. J Neurol 2004; 251(05):548-555

3 Albuquerque GCM, Guimarães BDA, Ribeiro MAS, Vaz RT, Silva JJX. Apresentação periorbitária de doença relacionada ao $\operatorname{IgG} 4$. Rev Bras Oftalmol 2018;77(05):286-288

4 Mikawa Y, Watanabe R, Hino Y, Hirano K. Hypertrophic spinal pachymeningitis. Spine 1994;19(05):620-625

5 Jimenez-Caballero PE, Diamantopoulos-Fernandez J, CamachoCastaneda I. [Hypertrophic cranial and spinal pachymeningitis. A description of four new cases and a review of the literature]. Rev Neurol 2006;43(08):470-475 